\title{
Estrés laboral y actividad física en empleados*
}

\section{Occupational stress and physical activity in employees}

\author{
Gustavo Ramón Suárez** \\ Universidad Cooperativa de Colombia - \\ Universidad de Antioquia \\ Medellin, Colombia \\ Santiago Zapata Vidales \\ Universidad Cooperativa de Colombia \\ Medellin, Colombia \\ Jaiberth Cardona-Arias \\ Universidad Cooperativa de Colombia - \\ Universidad de Antioquia \\ Medellin, Colombia
}

Recibido: 19 de junio de 2013 Revisado: 30 de agosto de 2013 Aceptado: 10 de octubre de 2013

\section{Resumen}

La actividad física puede proteger del estrés laboral. El objetivo del presente trabajo fue correlacionar la actividad física y el estrés laboral en trabajadores universitarios de Medellín, a partir de un estudio correlacional en 120 empleados seleccionados aleatoriamente y tamizados con International Physical Activity Questionnaire y el Test de Maslach-Jackson para el estrés. Se calcularon proporciones, chi cuadrado, intervalos para diferencias de medias y correlación de Spearman. Se halló sedentarismo en el $71 \%$, alto agotamiento emocional del $29 \%$, despersonalización del $38 \%$ y baja realización personal del $91 \%$. Se observó correlación inversa para la actividad física y el agotamiento emocional, y directa para despersonalización con agotamiento emocional y realización personal. Con esto resultados se concluyó que la inactividad física y el estrés laboral presentaron prevalencias elevadas; la actividad física presenta un efecto protector sobre el estrés.

Palabras clave: actividad física, agotamiento profesional, despersonalización, servicios de salud del trabajador, adulto joven.

* Artículo de investigación. Recursos en especie de la Universidad de Antioquia y la Universidad Cooperativa de Colombia sede Medellín

** Correspondencia: Jaiberth Antonio Cardona-Arias. Universidad de Antioquia, Universidad Cooperativa de Colombia. Dirección postal: Calle 67 No.53-108. Bloque 5, oficina 103. Medellín, Colombia. Correo electrónico: jaiberthcardona@gmail.com. 


\section{Abstract}

Physical activity is a protective factor for occupational stress. The objective in this study was to analyze the relationship between physical activity and occupational stress among universities employees of Medellin, through a relational study with 120 ramdomly selected employees. We used the International Physical Activity Questionnaire and the Maslach-Jackson test. Frequency, chi-square, confidence intervals for the mean difference and Spearman correlation were used for the analyses. Findings include sedentarism in $71 \%$ of the sample, high emotional exhaustion in $29 \%$, depersonalization in $38 \%$ and low personal accomplishment in $91 \%$. An inverse correlation was found between physical activity and emotional exhaustion, and positive for depersonalization with emotional exhaustion and personal accomplishment. With these results we concluded that physical inactivity and occupational stress have a high prevalence; physical activity has a protective effect on occupational stress.

Keywords: Motor activity, burnout, professional, depersonalization, occupational health services, young adult.

\section{Introducción}

La actividad física constituye un aspecto determinante de la calidad de vida. La Organización Mundial de la Salud (OMS) informa que la inactividad física es responsable de más de dos millones de muertes por año; el sedentarismo duplica el riesgo de enfermedades cardiovasculares, diabetes y obesidad y aumenta sustancialmente el riesgo de hipertensión arterial y otras enfermedades (Kalil, 2004). Frente a esto, la OMS propone la realización de actividad física de intensidad moderada mínimo 30 minutos, preferiblemente todos los días, los cuales pueden realizarse de forma fraccionada a lo largo del día en periodos de no menos de 10 minutos, y a partir de actividades cotidianas como caminar, subir escaleras, trabajar en el jardín o en las tareas de la casa.

Para la medición de la actividad física existe, entre otros instrumentos o escalas, el International Physical Activity Questionnaire IPAQ (Craig y Col., 2003), desarrollado en Ginebra en 1998 y validado en 12 países en el 2000, inclusive Colombia; este es válido y fiable para investigaciones sobre la prevalencia de actividad física en diferentes idiomas y países; cuenta con dos versiones, la corta que se emplea principalmente en sistemas de vigilancia nacional y regional, y la larga para obtener información más detallada en investigación o programas de evaluación (Booth, 2000).
El IPAQ ha sido usado en diversas investigaciones en Colombia, reportando una gran diversidad en las frecuencias de actividad física; así, en Bogotá se reportó 36,8 \% de actividad física regular (Gómez \& Col., 2005), en Teusaquillo estuvo entre $40 \%$ y 46 \% (Mantilla, 2006) y Prieto y Col., (2006) en Quindío, Bogotá y Antioquia, encontraron niveles insuficientemente activos en el $67 \%$ del grupo control, y en un $59 \%$ del grupo estudiado. Otros investigadores han referido variaciones en la frecuencia de actividad física según factores como la obesidad, el sexo y la escolaridad (Patiño \& Arango, 2011).

Aunado a la magnitud del problema, Martínez y Saldarriaga (2008) indican que la inactividad física aumenta la frecuencia y duración de las incapacidades laborales, esto supone implicaciones desfavorables para el trabajador, la empresa y la sociedad; por ello, los programas de promoción de la actividad física en trabajadores se plantean como una opción estratégica en salud ocupacional, particularmente para menguar el estrés laboral.

El estrés laboral es un desequilibrio entre las demandas del individuo y su capacidad de dar respuesta a ellas (McGrath, 1976). En este fenómeno, los riesgos psicososociales son los principales estresores, numerosos estudios han concluido que estos son agentes capaces de deteriorar la salud 
de las personas durante el desempeño de su trabajo e incluso fuera de él (Quick \& Tetrick, 2002). En Estados Unidos, el estrés laboral constituye un problema similar al de la Unión Europea. Según un informe del National Institute for Occupational Safety and Health (NIOSH, 1999), el porcentaje de trabajadores que informaron que su trabajo resultaba estresante estaba entre el $28 \%$ y el $40 \%$, en un estudio posterior (NIOSH, 2004, p. 34) se señala que los trabajadores afectados de ansiedad, estrés, o alteraciones neuróticas perdieron muchos más días de trabajo (25 días de promedio perdidos por trabajador) en el año 2001 que aquellos que no presentaban este tipo de alteraciones (solo 6 días como promedio perdidos por trabajador).

Desde la perspectiva clínica, Freudenberger (1974) empleó por vez primera el término Burnout para describir un conjunto de síntomas físicos sufridos por el personal sanitario como resultados de sus condiciones de trabajo. En 1981, Maslach y Jackson entienden que el Burnout se configura como "un síndrome tridimensional caracterizado por agotamiento emocional, despersonalización y reducida realización personal"; este puede ser medido a través del Maslach Burnout Inventory $(\mathrm{MBI})$, el cual presenta tres dominios: i) agotamiento emocional, para describir sentimientos como estar abrumado emocionalmente por el trabajo, ii) despersonalización, que describen una respuesta impersonal y falta de sentimientos hacia los sujetos objeto de atención, y iii) realización personal en el trabajo, para identificar sentimientos de competencia y realización exitosa en el trabajo. El MBI, elaborado inicialmente para profesionales de la salud, es fiable y válido para todo tipo de profesionales (García \& Col., 1992; Moreno y Col., 2000; Román, 2003; Fernández y Col., 2007).

Estudios previos han constatado la diversidad en la magnitud de este tópico, en 11.530 profesionales de la salud residentes en España y América Latina, la prevalencia de Burnout fue $14,9 \%$ en España, $14,4 \%$ en Argentina, 7,9 \% en Uruguay, 4,2 \% en México, 4 \% en Ecuador, 4,3\% en Perú, $5,9 \%$ en Colombia, $4,5 \%$ en Guatemala y $2,5 \%$ en El Salvador (Grau, Flichtentrei, Suñer, Prats,
Braga, 2009). Otros autores hallaron que para el caso de México fue del 35,5\% en docentes de escuela, (Unda, Sandoval, Gil-MonteGrau, 2008), para Chile un 27,4 \% en profesores de educación pre-escolar, básica o primaria y media o secundaria (Valdivia \& Col., 2003) y para Perú, un $40 \%$ en docentes de educación primaria y secundaria (Fernández, 2008).

Sumado a lo anterior, son múltiples los estudios que evidencian la relación entre la actividad física y el estrés, así: Hammer, Stamatakis y Steptoe (2009) hallaron una correlación de 0,59 (IC al $95 \%$ : 0.52-0.66; $p<0.001$ ) entre actividad física y la salud mental, y una reducción del estrés psicológico; Rimmele y Col. (2009) hallaron concentraciones significativamente más bajas de cortisol, de frecuencia cardiaca y estado de ansiedad en deportistas; Asztalos y Col. (2009) reportaron la asociación entre cinco tipos de actividad física (trabajo en casa, actividades de tiempo libre, andar en bicicleta desde la casa al trabajo, caminar desde la casa al trabajo, y practicar deportes) con dos dimensiones de la salud mental (percepción del estrés y el estrés psicológico); Toker y Biron (2012) encontraron una fuerte relación entre empleados con depresión y un bajo nivel de actividad física, y Cairney y Col., (2013) observaron que las personas que practican ejercicio vigoroso disminuyen el estrés y mejoran la salud general y su sentimiento de bienestar.

A pesar de la evidencia anterior, en nuestro medio son exiguos los estudios que investigan la relación entre estos dos tópicos, en una revisión de la literatura solo se halló un estudio en 201 trabajadores de áreas de la salud de una universidad de Medellín, en la cual se encontró que en personas con actividad física insuficiente el estrés laboral fue del $9,5 \%$ en contrate con los activos físicamente en quienes no se halló estrés laboral; además, en este grupo se registró una prevalencia de $40,8 \%$ para sobrepeso u obesidad y $6 \%$ síndrome de Burnout (Gómez, 2009).

Por lo expuesto, se realizó un estudio con el objetivo de analizar la relación entre el grado de actividad física y el estrés laboral en trabajadores de una universidad privada de Medellín. 


\section{Método}

Tipo de estudio: correlacional transversal.

Sujetos: 120 empleados de una universidad privada de Medellín, seleccionados mediante muestreo estratificado con asignación proporcional. El tamaño de muestra obedece a una población de referencia de 235 empleados, prevalencia del $16 \%$, confianza $95 \%$, error de muestreo $5 \%$ y corrección del $10 \%$ al muestreo.

Como criterios de inclusión estaban el tener contrato vigente, ser hombre o mujer de cualquier edad; en la exclusión se tuvo a quienes estuvieran bajo el efecto de alucinógenos o presentasen alteraciones cognitivas que pudiesen generar sesgo de información.

\section{Instrumentos}

Se utilizó como fuente de información primaria una encuesta individual y autodiligenciada, con variables sociodemográficas, el IPAQ versión corta y el test de estrés laboral de Maslach Burnout Inventory (Gil, 2002).

\section{Medición de la actividad física}

El IPAQ incluye las siguientes preguntas: i) durante los últimos 7 días, ¿cuántos días hizo usted actividades físicas vigorosas? ii) ¿Cuánto tiempo en total usualmente le toma realizar actividades físicas vigorosas en los días que las realiza? iii) ¿Cuántos días hizo usted actividades físicas moderadas? iv) ¿Cuánto tiempo en total usualmente le dedicó en uno de esos días que hizo actividades físicas moderadas? v) ¿Cuántos días caminó usted por lo menos 10 minutos seguidos? vi) ¿Cuánto tiempo en total pasó generalmente caminado en uno de esos días? Y, vii) durante los últimos 7 días, ¿cuánto tiempo en total usted usualmente pasó sentado durante un día en la semana?

El test clasifica el grado de actividad física en: i) alto, cuando se reporta una frecuencia semanal de actividad física igual a 7 , con combinación de caminata o actividades de moderada o alta in- tensidad logrando un mínimo de 3.000 MET-min/ semana. 0 cuando se reportó actividad vigorosa al menos 3 días a la semana alcanzando al menos $1.500 \mathrm{MET}$-min/semana; ii) moderado, cuando reporta 3 o más días de actividad vigorosa por al menos 20 minutos diarios; o cuando se reportaron 5 o más días de actividad moderada y/o caminata al menos 30 minutos diarios; o cuando se reportaron más de cinco días de cualquier combinación de caminata y actividades vigorosas o moderadas logrando al menos $600 \mathrm{MET}$-min/semana y iii) sedentario o bajo, cuando la frecuencia semanal de actividad física es menor o igual a un día, sin importar la intensidad ni la duración de la actividad.

\section{Medición del estrés laboral}

El test de Maslach y Jackson incluye 22 preguntas, nueve relacionadas con agotamiento emocional, cinco con despersonalización y ocho con realización personal y/o profesional. Cada pregunta presenta una escala Likert de 7 niveles (cero indica nunca y 6 todos los días), con base en estos se calculan puntajes de cada uno de los tres componentes los cuales se clasifican en alto (encima del percentil 75), medio (entre el percentil 75 y el 25) y bajo (por debajo del percentil 25).

\section{Plan de análisis}

Para describir el grupo de estudio, el grado de actividad física y los componentes del estrés laboral se calcularon frecuencias absolutas, relativas y medidas de resumen; para comparar el grado de actividad física según el sexo y la ocupación se utilizó la prueba Chi cuadrado de Pearson, mientras que su comparación según la edad se realizó mediante Anova; la comparación del agotamiento emocional, la despersonalización y la realización personal según el sexo y edad se realizó a través de las pruebas Chi cuadrado de Pearson, Fisher, Anova y t Student. La comparación del grado de actividad física y con la gradación de los tres componentes del estrés laboral se realizó por medio del coeficiente de correlación de Spearman. 
La elección de pruebas paramétricas y no paramétricas, se realizó con base en el cumplimiento o no del supuesto de normalidad bivariado evaluado con los estadísticos Kolomogorov Smirnov con corrección de la significación de Lilliefors y Shapiro Wilk, en Anova el supuesto de homocedasticidad se evaluó con el estadístico de Levene.

En todos los análisis se tomó una significación de 0,05 y éstos se realizaron en SPSS $21.0{ }^{\circledR}$.

\section{Aspectos éticos}

Se tuvieron presentes los principios de la Resolución 8430 y la Declaración de Helsinki. Prevaleció el respeto a la dignidad de los sujetos y la protección de sus derechos y privacidad, y se les informó el propósito, riesgos y beneficios del estudio. El proyecto tuvo aval del Comité de Bioética de la Universidad Cooperativa de Colombia.

\section{Resultados}

Se incluyeron personas con una edad promedio de 39,6 años, con un rango entre 19 y 69 , y el $50 \%$ de los valores centrales estuvo entre 32 y 48 años. En el grupo el 62 \% fueron mujeres, las principales ocupaciones incluidas fueron coordinador, secretaria y director; la prevalencia de sedentarismo fue $71 \%$, se presentó alto agotamiento emocional en el $29 \%$, despersonalización en el $38 \%$ y baja realización personal en el 91 \% (Tabla 1).

La frecuencia de actividad física no presentó asociación estadística con el género, la ocupación, la edad, la despersonalización ni la realización personal, pero si con el agotamiento emocional siendo mayor en las personas físicamente inactivas (Tabla 2). No obstante la ausencia de asociación estadística, llama la atención el elevado porcentaje $(29.2 \%)$ de agotamiento emocional en grado alto, especialmente en los hombres, donde fue del $37 \%$, frente al $24,3 \%$ en las mujeres; además, un gran porcentaje $(70,8 \%)$ de la muestra examinada presenta un bajo nivel de actividad física, con una ligera tendencia a tener mayores niveles en los hombres.
La edad no presentó asociación estadística con ninguno de los componentes del estrés laboral, mientras que el género solo presentó asociación con el agotamiento emocional siendo mayor el factor alto en los hombres (Tabla 3).

En la Tabla 4 se observa el análisis de los componentes del estrés laboral con la actividad física, en el grupo general y desagregado por sexo; se encontraron correlaciones estadísticamente significativas y directas entre la despersonalización y el agotamiento emocional, y entre la realización personal y la despersonalización para el grupo total y los hombres, y la actividad física se correlacionó negativamente con el agotamiento emocional.

La realización personal no presentó correlación estadísticamente significativa con la actividad física en el grupo $(\mathrm{Rho}=-0,009, \mathrm{Vp}=0,919) \mathrm{ni}$ al desagregar el análisis en los hombres (Rho= $-0,048, V p=0,749)$ ni en las mujeres (Rho $=-0,023$, $\mathrm{V} p=0,848)$.

\section{Discusión}

La prevalencia de inactividad física fue $71 \%$, alto agotamiento emocional $29 \%$, despersonalización 38 \% y baja realización personal $91 \%$; además se observó asociación estadística entre la actividad física y el agotamiento emocional, entre la despersonalización y el agotamiento emocional, y entre la realización personal y la despersonalización; esto pone de manifiesto la elevada magnitud del estrés laboral y el sedentarismo en los trabajadores de la universidad, y su interacción evidencia el efecto protector que podría tener la realización regular de alguna actividad física sobre el estrés laboral.

Con respecto a la actividad física, el $71 \%$ de la muestra examinada presentó un bajo nivel, siendo mayor la inactividad en mujeres. Tanto en el género masculino como el femenino se encontró un gran porcentaje con un bajo nivel de actividad física (65\% en hombres y $74 \%$ en mujeres); resultados que concuerdan con los encontrados en estudios previos en Colombia; así, Gómez y Col. (2005) hallaron solo un $36,8 \%$ de actividad física regular, 
y Mantilla (2006) entre el $40 \%$ y $46 \%$; mientras que Prieto y Col. (2006) encontraron niveles insuficientemente activos entre el $59 \%$ y $67 \%$.

En los dominios del estrés laboral, se observó un elevado porcentaje de agotamiento emocional, especialmente en los hombres, donde fue alto en $37 \%$, frente al $24,3 \%$ en las mujeres. En el factor despersonalización, en el género masculino se encontró un $37 \%$ en grado alto y en las mujeres $39 \%$. En el dominio de realización personal, se encontró un $91 \%$ en grado bajo para hombres y $90 \%$ para mujeres. Estos hallazgos evidencian que este síndrome afecta a todo tipo de personas trabajadoras en elevadas proporciones, e implica un gran reto para los prestadores de servicios de salud y los encargados de velar por el cuidado de la salud en el trabajo, en la medida que el estrés genera disminución y pérdida de recursos emocionales (la persona se vuelve menos sensible con los demás y muy duro consigo mismo), fatiga emocional, física y mental; deshumanización, insensibilidad, falta de realización personal, sentimientos de impotencia, inutilidad, baja autoestima y desesperanza, y desarrollo de una actitud negativa hacia el trabajo y la vida misma (Pines \& Kafry, 1978).

La magnitud del estrés laboral en el grupo de estudio es convergente con lo referido por otros estudios: Maslach y Jackson (1986) y la NIOSH (1999), quienes lo atribuyen a la cantidad de tareas en el trabajo y la desilusión gradual sobre el entorno (El Shali, 2010). Además, Freudenberger (1974), quien empleó por vez primera el término Burnout, alude que el estrés laboral se presenta con mayor frecuencia en los profesionales más comprometidos, quienes trabajan más intensamente ante la presión y demandas de su trabajo, que ponen en segundo término sus intereses, que son celosos en su trabajo y que realizan un sobreesfuerzo en su praxis laboral.

Con respecto a las posibles diferencias en la presentación del estrés entre hombres y mujeres, Duval, González y Rabia (2010) plantean que frente a este el organismo reacciona con un gran número de respuestas adaptativas que implican la activación del sistema nervioso simpático y el eje hipo- talámico-pituitario-adrenal, lo cual difiere según el sexo. Entre las causas de las manifestaciones agudas se encuentran: i) respuesta al estresor: el hombre activa preferentemente el cortex prefrontal, mientras que la mujer activa más bien el sistema límbico, ii) respuesta al estrés psicosocial: la reacción hipotalámica es más importante en el hombre que en la mujer, iii) implicación del estradiol en el control de la reactividad del eje HPA al estrés parece complejo, así como la influencia recíproca del eje HPA sobre el eje gonadotrópico.

En el estrés crónico la noción fundamental es que la hipercortisolemia es neurotóxica con relación a las estructuras cerebrales vulnerables como el hipocampo; así, las modificaciones cerebrales inducidas por el estrés (como la atrofia del hipocampo) tienen ramificaciones clínicas (depresión, diferencias individuales en el envejecimiento cerebral, etc.), aunque los procesos implicados no son más que parcialmente comprendidos en la actualidad.

En el actual estudio, se encontró una correlación inversa $(-0.320 p<0.001)$ entre nivel de actividad física y agotamiento emocional. Con respecto a dicha relación, Hamer, Stamatakis y Steptoe (2009), encontraron que con un mínimo de 20 minutos/ día/semana de actividad se producen beneficios mentales y que su aumento disminuye aún más el estrés psicológico. Rimmele y Col. (2009) al evaluar a 18 deportistas de élite, 50 deportistas y 24 personas no entrenadas, observaron que el grupo élite presentaba concentraciones significativamente más bajas de cortisol, de frecuencia cardiaca y estado de ansiedad que los sujetos desentrenados. Asztalos y Col. (2010) con sujetos entre los 25 y 64 años reportaron una asociación positiva entre actividad física y salud mental, siendo mayor en los hombres. Toker y Biron (2012) encontraron una fuerte relación entre la depresión e un grupo de empleados y la inactividad física. Donadio y Col. (2012) hallaron que las respuestas musculares a la excitación predicen la respuesta al estrés mental. Cairmey y Col. (2013), encontraron que quienes practican ejercicio vigoroso pueden disminuir el estrés y mejorar la salud general y su sentimiento de bienestar. 
Entre las principales limitaciones del estudio se encuentran el sesgo temporal y el carácter exploratorio de las asociaciones estadísticas. No obstante dichas limitaciones, este y otros estudios, ponen de manifiesto el efecto protector de la actividad física sobre los dominios del estrés laboral; además, se evidenció que la inactividad física y el estrés laboral presentan una elevada prevalencia en el grupo de estudio, lo que supone que los trabajadores incluidos en esta investigación presentan elevada carga de trabajo, compromiso, estrés, dificultades para invertir el tiempo libre en actividades físicas y otros factores que deben ser objeto de programas de atención y educación en salud y prevención de la enfermedad.

\section{Referencias}

Asztalos, M., Bourdeaudhuij, I. \& Cardon, G. (2010). The relationship between physical activity and mental health varies across activity intensity levels and dimensions of mental health among women and men. Public Health Nutrition, 13(8), 1.207-1.214.

Asztalos, M., Wijndaele, K., Bourdeaudhuij, I., Philippaerts, R., Matton, L., Duvigneaud, N., Thomis, M., Duquet, W., Lefevre, J. \& Cardon, G. (2009). Specific associations between types of physical activity and components of mental health. Journal of Science and Medicine in Sport, 12(4), 468-474.

Booth, M. L. (2000). Assessment of Physical Activity: An International perspective. Research quarterly for exercise and sport, 71(21), : s114-120.

Cairney, J., Kwan, M., Veldhuizen, S. \& Faulkner, G. (2013). Who uses exercise as a coping strategy for Stress? Results from a national survey of Canadians. J Phys Act Health. PMID: 23493043 (E-pub ahead of print).

Craig, C., Marshall, A., Sjöström, M., Bauman, A., Booth, M., Ainsworth, B., Pratt M., Ekelund, U., Yngve, A., Sallis, J. \& Oja, P. (2003). The Ipaq Consensus Group and the Ipaq Reliability and Validity Study Group. International Physical Activity Questionnaire (IPAQ): 12-country reliability and validity. Med Sci Sports Exerc, 35(13), 81-95.

Donadio, V., Liguori, R., Elam, M., Karisson, T., Giannoccaro, M., Pegenius, G., Giambattistelli, F. \& Wallin, B. G. (2012). Muscle sympathetic response to arousal predicts neurovascular reactivity during mental stress. The Journal of Physiology, 590(12), 2885- 2896.

Duval, F., González, F. \& Rabia, H. (2010). Neurobiología del estrés. Rev Chil Neuro-psiquiat, 48(4), 307-318.

El Sahili, L. F. (2010). Psicología para el Docente: consideraciones sobre los riesgos y desafíos de la práctica magisterial. Guadalajara, México: Universidad de Guanajuato.

Fernández, M. (2008). Burnout, Autoeficacia y Estrés en Maestros Peruanos: Tres Estudios Fácticos. Ciencia \& Trabajo, 10(30), 120-125.

Fernández-Lópeza, J. A., Fernández-Fidalgob, M., Martín-Payoc, R. \& Rödeld, A. (2007). Estrés laboral y calidad de vida en sanitarios de atención primaria: una prueba de la validez del cuestionario PECVEC. Aten Primaria, 39(8), 425-431.

Freudenberger, H. J. (1974). Staff Burnout. Journal of Social Issues, 30, 159-165.

García, M. \& Velandrino, A. P. (1992). EPB: Una escala para la evaluación del burnout profesional de las organizaciones. Anales de Psicología, 8(1-2), 131-138.

Gómez, A., García, S., Trujillo, D., Urrego, B., Ochoa, F. (2009). Obesidad, depresión, alcoholismo, estrés laboral y nivel de actividad física en una población laboral en el área de la salud de la ciudad de Medellín en el año 2009. Medellín: Universidad CES.

Gómez, L., Duperly, J., Lucumí, D., Gámez, R. \& Vanegas, A. (2005). Nivel de actividad física global en la población adulta de Bogotá 
(Colombia). Prevalencia y factores asociados. Gac Sanit, 19(3), 206-213.

Gil, P. (2002). Validez factorial de la adaptación al español del Maslach Burnout Inventory-General Survey. Salud Pública Méx, 44(1), 33-40.

Grau, A., Flichtentrei, D., Suñer, R., Prats, M. \& Braga, F. (2009). Influencia de factores personales, profesionales, y transnacionales en el Síndrome de Burnout en personal sanitario Hispanoamericano y Español (2007). Revista Española de Salud Pública, 83(2), 215-230.

Hammer, M., Stamatakis, E. \& Steptoe, A. (2009). Dose-response relationship between phscal activity and mental helath: The Scottish Health Surver. Br. J. Sports Med, 43, 1.1111.114 .

Kalil, M. (2004). Prevención de la hipertensión arterial: importancia de un estilo de vida saludable. Departamento de Hipertensión Arterial y Aterosclerosis. Belo Horizonte, Brasil: Servicio de Cardiología del Hospital Semper. Web de MSD. Recuperado de http://www.msd. com.co/msdco/jsp/hcp/diseases/hyper/articles/articulos.jsp?id=originalArticle_1614_es

Mantilla, S. (2006). Actividad física en habitantes de 15 a 49 años de edad de una localidad de Bogotá, Colombia. Rev. salud pública, 8(2), 69-80.

Martínez, E. \& Saldarriaga, J. F. (2008). Inactividad Física y Ausentismo en el Ámbito Laboral. Rev. salud pública, 10(2), 227-238.

Maslach, C. \& Jackson, S. E. (1986). MBI: Maslach Burnout Inventory. Manual. Palo Alto: University of California, Consulting Psychologists Press.

McGrath, J. E. (1976). Stress and behavior in organizations. In M. D. Dunnette (Ed.), Handbook of industrial and organizational psychology (pp. 1351-1395). Chicago: RandMcNally.

Moreno, B., Garrosa, E. \& González, J. L. (2000). Evaluación del estrés en el profesorado: el
CBP-R. Revista de psicología del trabajo y las organizaciones, 16(1), 331-349.

National Institute for Occupational Safety and Health NIOSH. (1999). Stress... at work. Publication No. 99-101. Cincinnati, OH: NIOSH. Recuperado de http://www.cdc.gov/niosh/ docs/99-101/pdfs/99-101.pdf

Patiño, F. A. \& Arango, E. F. (2011). Prevalencia de factores de riesgo cardiovascular y características de la práctica de la actividad física en la población entre 25 y 50 años de la zona urbana de municipio de Santa Rosa de Osos, Antioquia, 2009. Medellín: Funámbulos.

Pines, A. \& Kafry, D. (1978). Coping with burnout. Paper presented at the Annual. Toronto: Convention of the American Psychology Association.

Prieto, A. \& Agudelo, C. (2006). Enfoque multinivel para el diagnóstico de la actividad física en tres regiones de Colombia. Rev. salud pública, 8(2), 57-68.

Quick, J. C. \& Tetrick, L. E. (Eds.) (2002). Handbook of Occupational Health Psychology. Washington, D.C: American Psychological Association.

Rimmele, U., Seller, R., Marti, B., Wirtz. P., Ehlert, U. \& Heinrichs, M. (2009). The level of physical activity affects adrenal and cardiovascular reactivity to psychosocial stress. Psychoneuroendocrinology. 34(2), 190-198.

Román, H. J. (2003). Estrés y burnout en profesionales de la salud de los niveles primario y secundario de atención. Rev. Cubana de Salud Pública, 29(2), 103-110.

Toker, S. \& Biron, M. (2012). Job burnout and depression: Unravelun their temporal relationship and considering the role of physical activity. Journal of Applied Psychology, 97(3), 699-710.

Unda, S., Sandoval, J. \& Gil-MonteGrau, P. (2008). Prevalencia del síndrome de quemarse por el 
trabajo (SQT) (burnout) en maestros mexicanos. Información Psicológica, (91-92), 53-63.

Valdivia, G., Avendaño, C., Bastías, G., Milicic, N., Morales, A. \& Scharager, J. (2003). (PDF). Es- tudio de la salud laboral de los profesores en Chile: Informe Final de Proyecto. Santiago de Chile: Facultad de Medicina y Ciencias Sociales, Pontifica Universidad Católica de Chile.

Tabla 1.

Descripción del grupo de estudio y distribución porcentual del estrés laboral y la actividad física.

\begin{tabular}{|c|c|c|c|}
\hline & & $\#$ & $\%$ \\
\hline \multirow{2}{*}{ Género } & Masculino & 46 & 38,3 \\
\hline & Femenino & 74 & 61,7 \\
\hline \multirow{11}{*}{ Ocupación } & Administrador & 5 & 4,2 \\
\hline & Analista & 2 & 1,7 \\
\hline & Asesor & 5 & 4,2 \\
\hline & Asistente & 10 & 8,3 \\
\hline & Auxiliar & 12 & 10,0 \\
\hline & Coordinador & 35 & 29,2 \\
\hline & Director & 19 & 15,8 \\
\hline & Monitor & 3 & 2,5 \\
\hline & Secretaria & 23 & 19,2 \\
\hline & Subdirector & 3 & 2,5 \\
\hline & Técnico & 3 & 2,5 \\
\hline \multirow{3}{*}{ Actividad física } & Bajo & 85 & 70,8 \\
\hline & Medio & 14 & 11,7 \\
\hline & Alto & 21 & 17,5 \\
\hline \multirow{3}{*}{ Agotamiento emocional } & Bajo & 50 & 41,7 \\
\hline & Medio & 35 & 29,2 \\
\hline & Alto & 35 & 29,2 \\
\hline \multirow{3}{*}{ Despersonalización } & Bajo & 32 & 26,7 \\
\hline & Medio & 42 & 35,0 \\
\hline & Alto & 46 & 38,3 \\
\hline \multirow{3}{*}{ Realización personal } & Bajo & 109 & 90,8 \\
\hline & Alto & 11 & 9,2 \\
\hline & Media \pm DE & Mediana (RI) & Rango \\
\hline Edad & $39,6 \pm 9,9$ & $39,5(32,0-47,8)$ & $19,0-69,0$ \\
\hline
\end{tabular}

DE: Desviación estándar. RI: Rango intercuartil. 
Tabla 2.

Comparación de la actividad física según género, ocupación y estrés laboral.

\begin{tabular}{|c|c|c|c|c|c|c|c|}
\hline \multirow[b]{3}{*}{ Género } & \multicolumn{6}{|c|}{ Actividad física } & \multirow{3}{*}{$\mathrm{Vp} \mathrm{Chi}^{2}$} \\
\hline & \multicolumn{2}{|c|}{ Bajo } & \multicolumn{2}{|c|}{ Medio } & \multicolumn{2}{|c|}{ Alto } & \\
\hline & $\#$ & $\%$ & $\#$ & $\%$ & $\#$ & $\%$ & \\
\hline Masculino & 30 & 65,2 & 4 & 8,7 & 12 & 26,1 & \multirow{2}{*}{0,133} \\
\hline Femenino & 55 & 74,3 & 10 & 13,5 & 9 & 12,2 & \\
\hline \multicolumn{8}{|l|}{ Ocupación } \\
\hline Administrador & 3 & 60,0 & 0 & 0,0 & 2 & 40,0 & \multirow{11}{*}{0,064} \\
\hline Analista & 1 & 50,0 & 0 & 0,0 & 1 & 50,0 & \\
\hline Asesor & 4 & 80,0 & 1 & 20,0 & 0 & 0,0 & \\
\hline Asistente & 7 & 70,0 & 1 & 10,0 & 2 & 20,0 & \\
\hline Auxiliar & 8 & 66,7 & 1 & 8,3 & 3 & 25,0 & \\
\hline Coordinador & 26 & 74,3 & 4 & 11,4 & 5 & 14,3 & \\
\hline Director & 14 & 73,7 & 1 & 5,3 & 4 & 21,1 & \\
\hline Monitor & 1 & 33,3 & 1 & 33,3 & 1 & 33,3 & \\
\hline Secretaria & 18 & 78,3 & 5 & 21,7 & 0 & 0,0 & \\
\hline Subdirector & 3 & 100,0 & 0 & 0,0 & 0 & 0,0 & \\
\hline Técnico & 0 & 0,0 & 0 & 0,0 & 3 & 100,0 & \\
\hline \multicolumn{8}{|c|}{ Agotamiento emocional } \\
\hline Bajo & 27 & 54,0 & 8 & 16,0 & 15 & 30,0 & \multirow{3}{*}{$0,006^{* *}$} \\
\hline Medio & 28 & 80,0 & 2 & 5,7 & 5 & 14,3 & \\
\hline Alto & 30 & 85,7 & 4 & 11,4 & 1 & 2,9 & \\
\hline \multicolumn{8}{|c|}{ Despersonalización } \\
\hline Bajo & 25 & 78,1 & 4 & 11,4 & 1 & 2,9 & \multirow{3}{*}{0,215} \\
\hline Medio & 27 & 64,3 & 2 & 6,3 & 5 & 15,6 & \\
\hline Alto & 33 & 71,7 & 4 & 9,5 & 11 & 26,2 & \\
\hline \multicolumn{8}{|c|}{ Realización personal } \\
\hline Bajo & 77 & 70,6 & 13 & 11,9 & 19 & 17,4 & \multirow{2}{*}{0,962} \\
\hline \multirow[t]{2}{*}{ Alto } & 8 & 72,7 & 1 & 9,1 & 2 & 18,2 & \\
\hline & $\mathrm{X}$ & $\mathrm{DE}$ & $X$ & DE & $X$ & DE & Vp Anova \\
\hline Edad & 39,2 & 9,6 & 36,5 & 9,8 & 43,3 & 10,6 & 0,107 \\
\hline
\end{tabular}

X: Media. DE: Desviación estándar. ${ }^{* *}$ La asociación es significativa en el 0,01.

Tabla 3.

Comparación del estrés laboral según género y edad.

\begin{tabular}{|c|c|c|c|c|c|c|}
\hline \multirow{3}{*}{$\begin{array}{l}\text { Estrés laboral } \\
\text { Agotamiento emocional }\end{array}$} & \multicolumn{4}{|c|}{ Género } & \multicolumn{2}{|c|}{ Edad } \\
\hline & \multicolumn{2}{|c|}{ Masculino } & \multicolumn{2}{|c|}{ Femenino } & \multirow[t]{2}{*}{ Media } & \multirow[t]{2}{*}{ D. Estándar } \\
\hline & $\#$ & $\%$ & \# & $\%$ & & \\
\hline Bajo & 19 & 41,3 & 31 & 41,9 & 41,12 & 11,36 \\
\hline Medio & 10 & 21,7 & 25 & 33,8 & 36,77 & 7,94 \\
\hline Alto & 17 & 37,0 & 18 & 24,3 & 40,17 & 8,98 \\
\hline $\mathrm{Vp}$ & \multicolumn{4}{|c|}{$0,027^{a}$} & \multicolumn{2}{|c|}{$0,124^{c}$} \\
\hline \multicolumn{7}{|l|}{ Despersonalización } \\
\hline Bajo & 13 & 28,3 & 19 & 25,7 & 40,78 & 9,30 \\
\hline Medio & 16 & 34,8 & 26 & 35,1 & 38,12 & 10,24 \\
\hline Alto & 17 & 37,0 & 29 & 39,2 & 40,07 & 9,99 \\
\hline $\mathrm{Vp}$ & \multicolumn{4}{|c|}{$0,947^{a}$} & \multicolumn{2}{|c|}{$0,476^{c}$} \\
\hline \multicolumn{7}{|l|}{ Realización personal } \\
\hline Bajo & 42 & 91,3 & 67 & 90,5 & 39,43 & 9,780 \\
\hline Alto & 4 & 8,7 & 7 & 9,5 & 41,00 & 11,252 \\
\hline Vp & \multicolumn{4}{|c|}{$1,000^{\mathrm{b}}$} & \multicolumn{2}{|c|}{$0,618^{d}$} \\
\hline
\end{tabular}

a Prueba chi cuadrado de Pearson. ${ }^{\mathrm{b}}$ Prueba exacta de Fisher. ${ }^{\mathrm{C}}$ Anova. ${ }^{\mathrm{d}} \mathrm{T}$ Student. 
Tabla 4

Correlaciones de Spearman para el grado de actividad física y los dominios del estrés.

\begin{tabular}{lcccccc}
\hline & \multicolumn{2}{c}{ Total } & \multicolumn{2}{c}{ Hombre } & \multicolumn{2}{c}{ Mujer } \\
\hline & A. E. & Desp. & A. E. & Desp. & A. E. & Desp. \\
\hline Despersonalización & $0,353^{* *}$ & & $0,570^{* *}$ & & 0,200 & \\
Realización personal & 0,116 & $0,211^{*}$ & $0,362^{*}$ & $0,358^{*}$ & $-0,039$ & 0,121 \\
Actividad Física & $-0,320^{* *}$ & 0,013 & $-0,351^{*}$ & $-0,068$ & $-0,320^{* *}$ & 0,081 \\
\hline
\end{tabular}

A.E. Agotamiento emocional. Desp. Despersonalización. * La correlación es significativa en el 0,05. " La correlación es significativa en el 0,05. 